ACCEPTED MANUSCRIPT

\title{
Determining the Layers' Young's Moduli and Thickness from the Indentation of a Bilayer Structure
}

To cite this article before publication: Yin Zhang et al 2018 J. Phys. D: Appl. Phys. in press https://doi.org/10.1088/1361-6463/aaa55d

\section{Manuscript version: Accepted Manuscript}

Accepted Manuscript is "the version of the article accepted for publication including all changes made as a result of the peer review process, and which may also include the addition to the article by IOP Publishing of a header, an article ID, a cover sheet and/or an 'Accepted Manuscript' watermark, but excluding any other editing, typesetting or other changes made by IOP Publishing and/or its licensors"

This Accepted Manuscript is @ 2018 IOP Publishing Ltd.

During the embargo period (the 12 month period from the publication of the Version of Record of this article), the Accepted Manuscript is fully protected by copyright and cannot be reused or reposted elsewhere.

As the Version of Record of this article is going to be / has been published on a subscription basis, this Accepted Manuscript is available for reuse under a CC BY-NC-ND 3.0 licence after the 12 month embargo period.

After the embargo period, everyone is permitted to use copy and redistribute this article for non-commercial purposes only, provided that they adhere to all the terms of the licence https://creativecommons.org/licences/by-nc-nd/3.0

Although reasonable endeavours have been taken to obtain all necessary permissions from third parties to include their copyrighted content within this article, their full citation and copyright line may not be present in this Accepted Manuscript version. Before using any content from this article, please refer to the Version of Record on IOPscience once published for full citation and copyright details, as permissions will likely be required. All third party content is fully copyright protected, unless specifically stated otherwise in the figure caption in the Version of Record.

View the article online for updates and enhancements. 


\title{
Determining the layers' Young's moduli and thickness from the indentation of a bilayer structure
}

\author{
Yin Zhang ${ }^{a, b}$, Ya-pu Zhao ${ }^{a, b}$ and Zhihai Cheng ${ }^{c}$ \\ ${ }^{a}$ State Key Laboratory of Nonlinear Mechanics (LNM), Institute of Mechanics, Chinese Academy of Sciences, Bei- \\ jing 100190, China. \\ ${ }^{b}$ School of Engineering Science, University of Chinese Academy of Sciences, Beijing 100049, China. \\ ${ }^{c}$ Department of Physics and Beijing Key Laboratory of Optoelectronic Functional Materials \& Micro-Nano Devices,
} Renmin University of China, Beijing 100872, China.

Abstract The inverse problem of determining three parameters: the film thickness, the film and substrate Young's moduli of a film/substrate bilayer by indentation, is formulated and solved. The physical mechanism for the solvability of the inverse problem is that these three parameters have different impacts at different indentation depth. Their impacts are systematically studied, which also provides a different approach of finding the three parameters or refining their range. Compared with various atomic force microscopy (AFM) based techniques of detecting subsurface structures, which have to deal with an extremely difficult or even an insurmountable inverse problem with the integral equation of dynamics, the inverse problem here formulated by statics is much more straightforward and simpler. Formulating and solving such inverse problem can be of some help to the applications such as characterizing subsurface structures, the out-of-plane properties of two-dimensional (2D) materials, and various bilayer structures.

\section{Introduction}

Characterizing subsurface structure is critical to almost every area of science and engineering [1]. For example, one of crucial demands in microelectronics is to detect the integrity of electrical contacts and interconnects, which are often buried and stacked [2]. Detecting the inner structure of biological sam- 
ples can help to identify the presence of malaria parasites [2] or nanoparticles [3] inside a red blood cell. Probing the subsurface ionic transport is key to the development of various energy storage and generation systems $[4,5]$. To detect the subsurface structure, various atomic force microscopy (AFM) based dynamic techniques are developed, such as ultrasonic force microscopy (UFM) [6], atomic force acoustic microscopy (AFAM) [7], heterodyne force microscopy (HFM) [8], scanning near-filed ultrasound holography (SNFUH) [2], multifrequency force microscopy [9], resonant/difference-frequency atomic force ultrasonic microscopy (RDF-AFUM) [10] and mode synthesizing atomic force microscopy (MSAFM) [11], etc. The buried structures, interfaces, defects and particles can all lead to the mechanical property variations such as stiffness, elasticity, viscosity and adhesion energy [2, 12], which are manifested by the AFM tip-sample interactions. Those mechanical property variations are characterized by three observable parameters of an AFM oscillation: amplitude, frequency and phase lag [12]. In the layered film/substrate structures $[13,14,15]$ or the structures with buried particles and defects [16], the change of AFM tip indentation depth leads to the variations of radiation impedance and contact stiffness, which both result in the shifts of the AFM contact resonance frequencies. Therefore, the shifts of the contact resonance frequencies are used to infer the mechanical properties of subsurface layer, particles and defects. Similarly, phase lag, which indicates the energy dissipation, can also be used to image/detect the subsurface structures [12].

However, for those AFM-based dynamic techniques to detect the mechanical properties of subsurface structures, there are two major technical challenges in the interpretation of AFM oscillation parameters [17]: Firstly, it is not straightforward and even extremely difficult to link the observable AFM oscillation parameters of amplitude, frequency and phase lag with the specific information about the subsurface structure. The second one is how to separate topography from mechanical properties in those parameters. These two interpretation challenges in essence are due to the mathematical difficulty 
of solving an inverse problem $[18,19,20]$. In order to determine the sample properties, the inverse problem of extracting the tip-sample interactions from the measured motions of the probe needs to be solved, which is an integral equation $[18,19,20]$. In the integral equation of AFM dynamics, the interaction force/potential is often multiplied/divided by the instantaneous tip displacement or its derivatives. Mathematically, it is very difficult or even impossible to invert such integral equation, or say, to extract the interaction force/potential as a function of the measured experimental parameters [9]. The inverse problem of the AFM dynamic tip-sample interactions in general is unsolvable without making some assumptions to simplify the problem $[18,19,20]$. Although various AFM dynamic techniques as mentioned above are developed and much more information on the AFM oscillations can be measured, it is still a significant challenge to transform the myriad of information into the specific knowledge about the subsurface structural and mechanical properties [17]. As a result, the current dynamic AFM techniques detect the subsurface structures in a qualitative rather than a quantitative way [12].

In the film/substrate bilayer indentation tests $[21,22,23,24,25]$, the elastic field is not confined to the film layer only and it also extends into the substrate layer. Therefore, the substrate properties play a role in the indentation test, which is the so-called substrate effect. In those indentation tests $[21,22,23,24,25]$, the substrate effect is hazardous because it causes significant troubles of accurately measuring the film properties. In contrast, the substrate effect is the exact mechanism for those AFM dynamic techniques $[13,14,15,16]$ to detect the mechanical properties of a subsurface layer. On the other hand, various two-dimensional (2D) materials are combined together by stacking to form heterogeneous structures $[26,27,28]$, which demonstrate some unique and excellent properties because of their anisotropy [29]. One remarkable characteristics of the $2 \mathrm{D}$ material anisotropy is that its in-plane elasticity is an intrinsic property, while, the out-of-plane counterpart is a tunable one [29]. The reason for such tunability is that it is the weak van der Waals (vdW) force linking the layers [27] and ions/molecules can be easily inserted

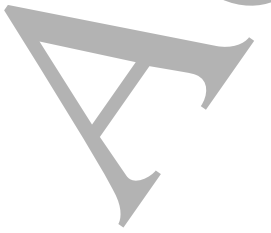


between the layers [28], which essentially changes the vdW force and thus the 2D material out-of-plane properties. Characterizing the 2D material out-of-plane properties, such as Young's modulus, is essential for both fundamental understanding and practical applications [29]. In contrast to various measuring methods on the in-plane Young's modulus of 2D material, such as bending and axial tensile test [30, 31], the indentation test may be the only technique currently available to measure the $2 \mathrm{D}$ material out-ofplane properties [32]. Gao's method of measuring the out-of-plane Young's modulus of 2D materials in essence is to avoid/neglect the substrate effect [32]. In their graphene/silicon carbide (SiC) bilayer indentation test [32], the indentation depth is $0.1 \mathrm{~nm}$ and the graphene is ten-layer with the total (estimated) thickness of $3 \mathrm{~nm}$. Therefore, Gao et al. [32] assumed that the SiC substrate has no impact on the indentation. To avoid the substrate effect and thus to measure the "film-only" properties, the common practice is to limit the indentation depth less than $10 \%$ of the film thickness [24]. However, this $10 \%$ ratio rule of thumb is actually not reliable [22]. Even when the ratio of the indentation depth to the film thickness is as small as 1\%, the substrate effect can still be significant [33]. The ratio in Gao's indentation test [32] is $0.1 / 3 \approx 3.33 \%$ and as shown later, the substrate effect stands out even more when the Young's modulus difference between the film and substrate is larger. Besides a $2 \mathrm{D}$ material on a substrate, the film/substrate model also applies to various coated materials with a bilayer structure [33, 34].

In the above film/substrate bilayer indentation tests $[21,22,23,24,25]$, which intends to measure the film Young's modulus only, both the film thickness and substrate Young's modulus are the known quantities. As a result, the film Young's modulus as the only unknown can be uniquely determined because of the one-to-one relation between the film Young's modulus and the film/substrate effective Young's modulus $[21,22,23,24,25]$. The film/substrate effective Young's modulus is a quantity varying with indentation depth, which can be extracted from the force-indentation depth curves [21, 22, 23, 24, 25]. However, for those AFM dynamic techniques to detect the structure of subsurface layer [13, 14], the film 
thickness and substrate Young's modulus are also unknown. When the number of unknown quantity is more than one, the above one-to-one relation does not exists and as shown later, there are infinite combinations of those unknown quantities which can result in a same film/substrate effective Young's modulus. This is also the mathematical reason for the difficulty of solving the inverse problem. Batog et al. [15] even concluded that only when either two of the film thickness, the film and substrate Young's moduli are known, can a third one be determined. In this study, the film thickness, the film and substrate Young's moduli are the three unknowns to be determined as an inverse problem from the indentation data. Physically, the inverse problem in this study is formulated via the statics of indentation. Compared with the above AFM dynamic techniques, this statics formulation provides a simpler and more straightforward method to detect the subsurface structures together with the surface ones. In those AFM dynamic techniques utilizing the shifts of contact resonance to detect the subsurface structures $[13,14,16]$, the contact stiffness and damping are the two varying quantities determining the contact resonance. In our statics formulation of inverse problem, the contact stiffness is de facto the only varying quantity, from which the film/substrate effective/Young's modulus is extracted. Although our inverse solving method can determine the three unknowns of the film/thickness, the film and substrate Young's moduli, its accuracy is strongly dependent on that of the indentation measurements. To overcome this robustness issue, a systematic approach to improve the accuracy is also presented. 


\section{Model development}

The contact models of Hertz, Derjaguin-Muller-Toporov (DMT) and Johnson-Kendall-Roberts (JKR) are given as follows

$$
P=\left\{\begin{array}{l}
\frac{4 E^{*} a^{3}}{3 R}, \quad(\text { Hertz }) \\
\frac{4 E^{*} a^{3}}{3 R}-2 \pi R \gamma, \quad(\mathbf{D M T}) \\
\frac{4 E^{*} a^{3}}{3 R}-2 a^{3 / 2} \sqrt{2 \pi E^{*} \gamma},
\end{array}\right.
$$

$($ JKR).

Where $P, a$ and $\gamma$ are the indentation load, the contact radius and the work of adhesion between the indenter and film surfaces, respectively. $1 / R=1 / R_{i}+1 / R_{f}\left(R_{i}\right.$ : indenter radius and $R_{f}$ : the film radius of curvature). $E^{*}$ is the reduced modulus of the specimen/indenter system, which is given as the following

$$
\frac{1}{E^{*}}=\frac{1}{E^{\prime}}+\frac{1}{E_{i}^{\prime}}
$$

As shown in figure 1, the specimen is a bilayer structure of film and substrate. Here $E^{\prime}$ is the reduced modulus of the specimen and its expression will be discussed later in details. $E_{i}^{\prime}=E_{i} /\left(1-\nu_{i}^{2}\right)$ is the reduced modulus of indenter with $E_{i}$ and $\nu_{i}$ being the indenter Young's modulus and Poisson's ratio, respectively. It is also noticed that the reduced modulus and the plane-strain modulus are the same [25], which physically indicates that the contact stress is triaxial with a high degree of hydrostatic pressure [21].

Eq. (1) presents the $a-P$ curves. However, the contact radius $(a)$ is not a measured quantity by a depth-sensing indentation instrument $[24,23,35,36]$. The following relation holds for the contact depth $\left(h_{c}\right)$ and radius $(a)[37,38]$

$$
h_{c}= \begin{cases}\frac{a^{2}}{R}, & (\text { Hertz }, \text { DMT }) \\ \frac{a^{2}}{R}-\sqrt{\frac{2 \pi R \gamma}{E^{*}}}, & (\text { JKR }) .\end{cases}
$$

Because the layer elastic and plastic deformations can result in sink-in and pile-up, respectively, the contact depth $\left(h_{c}\right)$ and measured indentation depth $(h)$ are often different $[24,35,39]$ In the sink-in 


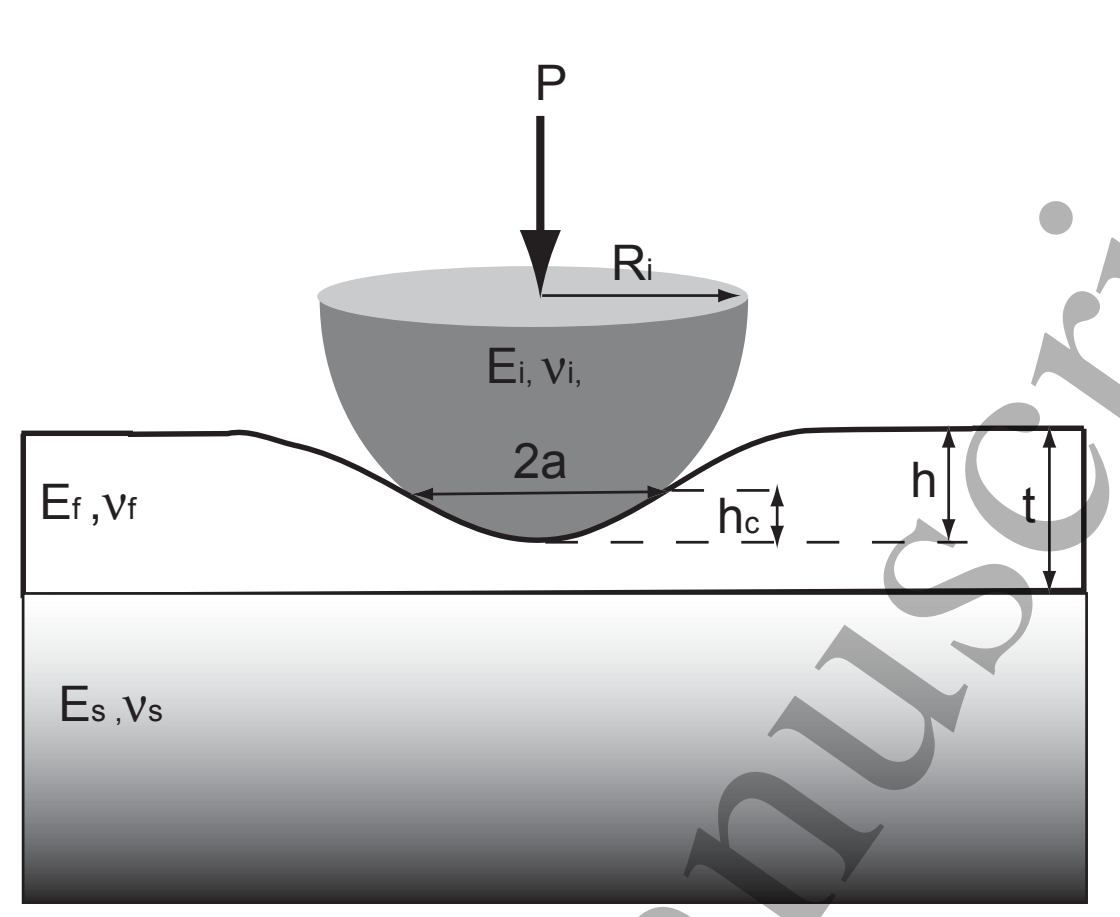

Figure 1. Schematic diagram of an indenter penetration into a film/substrate structure. The indenter is with the radius of $R_{i}$, Young's modulus of $E_{i}$ and Poisson's ratio of $\nu_{i} . t$ is the film thickness, $h_{c}$ and $a$ are the contact depth and radius, respectively. $P$ is the indentation load and $h$ is the indenter displacement. $E_{f}, \nu_{f}$ and $E_{s}, \nu_{s}$ are the Young's moduli and Poisson's ratios of the film and substrate, respectively.

scenario as shown in figure $1, h_{c}<h$; while, in the pile-up scenario, $h_{c}>h$ [34]. For simplicity, $h=h_{c}$ is assumed here, which can cause an error. The impact of this error together with others will be discussed later in details.

With both Eqs. (1) and (3), the $h-P$ curve can be easily obtained. However, in real applications, the work of adhesion $(\gamma)$ measured by pull-off forces fluctuates significantly [32], which can cause significant errors on the $h-P$ curve interpretation. To lessen this adhesion influence, the contact stiffness $(K)$ is introduced as follows by taking the derivative of $d P / d h_{c}[32]$

$$
K=\frac{d P}{d h_{c}}=\frac{d P / d a}{d h_{c} / d a}= \begin{cases}2 E^{*} a=2 E^{*} \sqrt{R h_{c}}, & (\text { Hertz }, \text { DMT }) \\ \frac{4 E^{*} a^{2} / R-3 \sqrt{2 \pi a \gamma}}{2 a / R-\sqrt{\pi \gamma /\left(2 E^{*} a\right)}}, & (\text { JKR }) .\end{cases}
$$


In above equation, $\gamma$ has no impact on the Hertz and DMT models. When $\gamma=0$, $d P / d h_{c}$ of the JKR models recovers that of the Hertz and DMT models. The derivation of Eq. (4) solely relies on elasticity. While, plastic deformation often occurs in an indentation [24, 35, 36]. In/order to incorporate the plasticity effect, the following modified version of Eq. (4) is introduced [39];

$$
K=C \frac{2}{\sqrt{\pi}} E^{*} \sqrt{S_{A}}
$$

Where $C$ is a dimensionless correction factor to account for the effect of the indenter geometry. For example, $C=1$ for sphere indenter; $C=1.012$ for square indenter (punch)/and $C=1.034$ for triangular indenter [36]. $S_{A}=\pi a^{2}$ is the projected contact area and in a real application, the proper calculation/estimation of $S_{A}$ can be difficult [40]. The difficulty is due to the $h_{c}$ and $h$ difference as mentioned above. Because $a$ is not a measured quantity, in order to calculate $S_{A}$, the $h_{c}-a$ relation of Eq. (3) needs to be used. While, because of the sink-in and pile-up effects, it can be difficult to obtain $h_{c}$ accurately from the measured data of $h$. Clearly, when $C=1$, Eq. (5) recovers Eq. (4) of the Hertz and DMT models.

In an indentation test, $P, h, R$ and $\gamma$ are the measured quantities. The contact radius $a$ is calculated from Eq. (3). The contact stiffness $K=K(a)$ or $K=K(h)$ varying with contact radius/depth is calculated from the measured $h-P$ data. Once $K$ is found, the reduced modulus of the specimen/indenter system $\left(E^{*}\right)$ can be determined. For example, $E^{*}=K /\left(2 \sqrt{R h_{c}}\right)$ can be easily derived by using the DMT model of Eq. (4). With $E^{*}$ being found, the reduced modulus of the specimen $\left(E^{\prime}\right)$ is determined from Eq. (2) as follows:

$$
E^{\prime}=\frac{E^{*} E_{i}^{\prime}}{E_{i}^{\prime}-E^{*}}
$$

Here the indenter reduced modulus of $E_{i}^{\prime}$ is a known quantity. $E^{\prime}$ is a function of the reduced moduli of the film and substrate $\left(E_{f}^{\prime}\right.$ and $\left.E_{s}^{\prime}\right)$, the film thickness $(t)$ and contact radius or depth $(a$ or $h)$, i.e., $E^{\prime}=E^{\prime}\left(E_{f}^{\prime}, E_{s}^{\prime}, t, a\right)$ or $E^{\prime}=E^{\prime}\left(E_{f}^{\prime}, E_{s}^{\prime}, t, h\right)$. By examining various function forms given for $E^{\prime}=$ 
$E^{\prime}\left(E_{f}^{\prime}, E_{s}^{\prime}, t, a\right)$, Menčik et al. [21] found that only two function forms can properly fit the experimental data. One is the following exponential function form:

$$
E^{\prime}=E_{s}^{\prime}+\left(E_{f}^{\prime}-E_{s}^{\prime}\right) e^{-\Lambda a / t}
$$

Where $\Lambda$ is a positive fitting parameter, which can be obtained from the experimental data by the least square method [21]. The other is the following form [41]

$$
E^{\prime}=E_{s}^{\prime}+\left(E_{f}^{\prime}-E_{s}^{\prime}\right) \phi_{g}(x)
$$

Where $x=a / t$ is a dimensionless quantity, and $\phi_{g}(x)$ is a function given as follows [21, 41]

$$
\phi_{g}(x)=\frac{2}{\pi} \arctan \frac{1}{x}+\frac{1}{2 \pi(1-\nu)}\left[(1-2 \nu) \frac{\ln \left(1+x^{2}\right)}{x}-\frac{x}{1+x^{2}}\right]
$$

Here $\nu$ is the effective Poisson's ratio, whose value depends on the contact radius and film thickness, i.e. $\nu=\nu(a / t)$. This $\nu$ value transitions from the film Poisson's ratio $\left(\nu_{f}\right)$ to the substrate one $\left(\nu_{s}\right)$ with the increase of contact radius/depth $(a$ or $h)$ [41]. Because the impact of this $\nu$ variation on the $E^{\prime}$ value as given by Eq. (8) is rather small [42], Menčik et al. suggested to simply use $\nu_{f}$ for $\nu$ [21]. Compared with Eq. (7), an outstanding characteristics of Eq. (8) is that it has no fitting parameter. Both $e^{-\Lambda x}$ and $\phi_{g}(x)$ monotonically decrease from 1 to 0 as $x$ increases from 0 to $\infty$, the $E^{\prime}$ value changes from $E_{f}^{\prime}$ to $E_{s}^{\prime}$ as indicated by both Eqs. (7) and (8). Physically this is the indication that the substrate effect enlarges with the increase of contact radius/depth. In both Eqs. (7) and (8), the contact radius $a$ is used and therefore, the measured quantity of $h_{c}$ needs to be transformed into a by using Eq. (3).

The following quantities are introduced for the nondimensionalization scheme:

$$
A=\frac{a}{a_{c}}, \quad F=\frac{P}{P_{c}}, \quad \alpha_{1}=\frac{E^{*}}{E_{i i}^{\prime}}, \quad \alpha_{2}=\frac{E_{f}^{\prime}}{E_{i i}^{\prime}}, \quad \alpha_{3}=\frac{E_{s}^{\prime}}{E_{i i}^{\prime}}, \quad \beta_{1}=\frac{a_{c}}{R}, \quad \beta_{2}=\frac{2 \pi \gamma}{E^{*} a_{c}}=\frac{2 \pi \gamma}{\alpha_{1} E_{i i}^{\prime} a_{c}}, \quad T=\frac{t}{a_{c}}, \quad x=\frac{a}{t}=\frac{A}{T}
$$

Here $E_{i i}^{\prime}=E_{i}^{\prime} / 2$ (i.e., $\left.1 / E_{i i}^{\prime}=1 / E_{i}^{\prime}+1 / E_{i}^{\prime}\right)$ is the reduced modulus as if the indenter is in contact with a same monolithic material; $a_{c}=\left[9 \pi \gamma R /\left(8 E_{i s}^{\prime}\right)\right]^{1 / 3}$ and $-P_{c}=-3 \pi R \gamma / 2$ are the pull-off radius and force 
as predicted by the JKR model. Eqs. (3) and (4) are now nondimensionalized as follows:

$$
H=\frac{h_{c}}{a_{c}}=\left\{\begin{array}{lc}
\beta_{1} A^{2}, & (\text { Hertz }, \text { DMT }) \\
\beta_{1} A^{2}-\sqrt{\beta_{2} A}, & (\text { JKR })
\end{array}\right.
$$

and

$$
S=\frac{K a_{c}}{P_{c}}= \begin{cases}\frac{3 \alpha_{1}}{2 \beta_{1}} A=\frac{3 \alpha_{1}}{2 \beta_{1}} \sqrt{\frac{H}{\beta_{1}}}, & (\text { Hertz, DMT) } \\ \frac{3 \alpha_{1} A^{2}-3 \sqrt{\alpha_{1}} A^{1 / 2}}{2 \beta_{1} A-1 / 2 \sqrt{\beta_{2} / A}}=\frac{3 \alpha_{1} A^{5 / 2}-3 \sqrt{\alpha_{1}} A}{2 \beta_{1} A^{3 / 2}-1 / 2 \sqrt{\beta_{2}}}, & \text { (JKR) }\end{cases}
$$

Eqs. (7) and (8) are nondimensionalized together as follows:

$$
\frac{E^{\prime}}{E_{i i}^{\prime}}=f=\alpha_{3}+\left(\alpha_{2}-\alpha_{3}\right) \phi(x)
$$

Where $\phi(x)$ can be either the exponential function of $\phi(x)=e^{-\Lambda x}$ as given by Eq. (7) or $\phi(x)=\phi_{g}(x)$ as given by Eq. (8). Actually, the difference between $e^{-\Lambda x}$ and $\phi_{g}(x)$ is very small. For example, with $\Lambda=0.8$ and in the range of $10^{-4} \leq x \leq 1.4$, the difference is less than $5 \%$ [21]. It should keep in mind that Eq. (14) in essence is an approximate expression. More accurate and strict elasticity analysis involves solving the integral equation of a Boussinesq problem [43]. However, even with the simplified expression of Eq. (14), significant mathematical difficulty as shown later can still arise because of the overdetermined property of solving the inverse problem of indentation [44].

The following equation is derived by equating Eqs. (13) and (14):

$$
\mathcal{F}\left(\alpha_{1}, A, \alpha_{2}, \alpha_{3}, T\right)=\frac{2 \alpha_{1}}{2-\alpha_{1}}-\alpha_{3}-\left(\alpha_{2}-\alpha_{3}\right) \phi(x)=0
$$

Here the dimensionless reduced modulus of specimen/indenter system $\left(\alpha_{1}\right)$ and dimensionless contact radius $(A)$ are the two varying and measured quantities. The dimensionless reduced moduli of film $\left(\alpha_{2}\right)$ 
and substrate $\left(\alpha_{3}\right)$, and the dimensionless film thickness $(T)$ are the three fixed but unknown parameters to be determined. Eq. (15) is the very foundation for us to solve the inverse problem.

\section{Results and discussion}

The following parameters are all taken from Gao's experimental data [32]: The indenter is made of silicon with the Young's modulus of $E_{i}=169 \mathrm{GPa}$ and the Poisson's ratio of 0.27 ; the indenter radius is fixed as $R_{i}=114 \mathrm{~nm}$ and the film/substrate bilayer is flat, which leads to $R=114 \mathrm{~nm}$; the work of adhesion is fixed as $\gamma=1 \mathrm{Nm}^{-1}$. As a result, $a_{c}$ and $\beta_{1}$ are fixed as $a_{c}=7.96 \mathrm{~nm}$ and $\beta_{1}=6.9828 \times 10^{-2}$. For silicon carbide $(\mathrm{SiC}), E_{S i C}=400 \mathrm{GPa}$ and $v_{S i C}=0.14$; for germanium $(\mathrm{Ge}), E_{G e}=138 \mathrm{GPa}$ and $v_{G e}=0.26$. In figure 2 , there are two types of film/substrate bilayer structures: $\mathrm{SiC} / \mathrm{Ge}$ (harder film) and $\mathrm{Ge} / \mathrm{SiC}$ (softer film). The $\mathrm{SiC} / \mathrm{Ge}$ bilayer corresponds to $\alpha_{2}=4.4764$ and $\alpha_{3}=1.6239 ; \mathrm{Ge} / \mathrm{SiC}$ corresponds to $\alpha_{2}=1.6239$ and $\alpha_{3}=4.4764$. In figure 2 , the $H-S$ curves as predicted by both JKR and DMT/Hertz models are plotted and the dimensionless film thickness is $T=2$. The DMT and Hertz models predict the same contact stiffness as given by Eq. (4) or (12). Adhesion has no contribution to the contact stiffness as predicted by the DMT and Hertz models. With the increase of indentation depth $(H)$, the contact stiffness difference between the JKR and DMT/Hertz models shrinks as seen in figure 2. Because the adhesion energy is a small quantity, the adhesion effect on contact needs to be considered only when the contact radius is very small [42]. It is also noticed that around $H=0.2$, the $H-S$ curves of $\mathrm{SiC} / \mathrm{Ge}$ and $\mathrm{Ge} / \mathrm{SiC}$ cross each other. The inset of figure 2 shows the $H-S$ curves as predicted by the DMT model in the shallow indentation area. For $H>0.2$, the contact stiffness of $\mathrm{SiC} / \mathrm{Ge}$ is smaller than that of $\mathrm{Ge} / \mathrm{SiC}$; for $0 \leq H<0.2$, it is the opposite: $\mathrm{SiC} / \mathrm{Ge}$ is with a larger contact stiffness and $\mathrm{Ge} / \mathrm{SiC}$ is with a smaller one. To illustrate the crossing mechanism of $H-S$ curves, figure 3 is presented. Figure 3 plots the $f$ as a function of $H$ with $T=2$. Here $f=E^{\prime} / E_{i i}^{\prime}$ as defined in both Eqs. (13) and 
(14) is the dimensionless reduced modulus of the film/substrate. As mentioned above, because of the monotonically decreasing property of $e^{-\Lambda x}$ and $\phi_{g}(x)$, the $f$ value experiences the transition from the film one $\left(\alpha_{2}\right)$ to the substrate one $\left(\alpha_{3}\right)$ with the increase of $H$. In figure 3 , for $\mathrm{SiC} / \mathrm{Ge}$, it is a harder film on a softer substrate; $f$ starts as $f=\alpha_{2}=4.4764$ and decreases monotonically towards $\alpha_{3}=1.6239$. While, for $\mathrm{Ge} / \mathrm{SiC}$, it is the softer film on harder substrate case, $f$ starts as $f=\alpha_{2}=1.6239$ and then increases monotonically towards $\alpha_{3}=4.4764$. The two curves also cross each other exactly at $H=0.2$. The change of the reduced modulus of film/substrate [45], or say, the substrate effect, is the mechanism responsible for the crossings in both figures 2 and 3 .

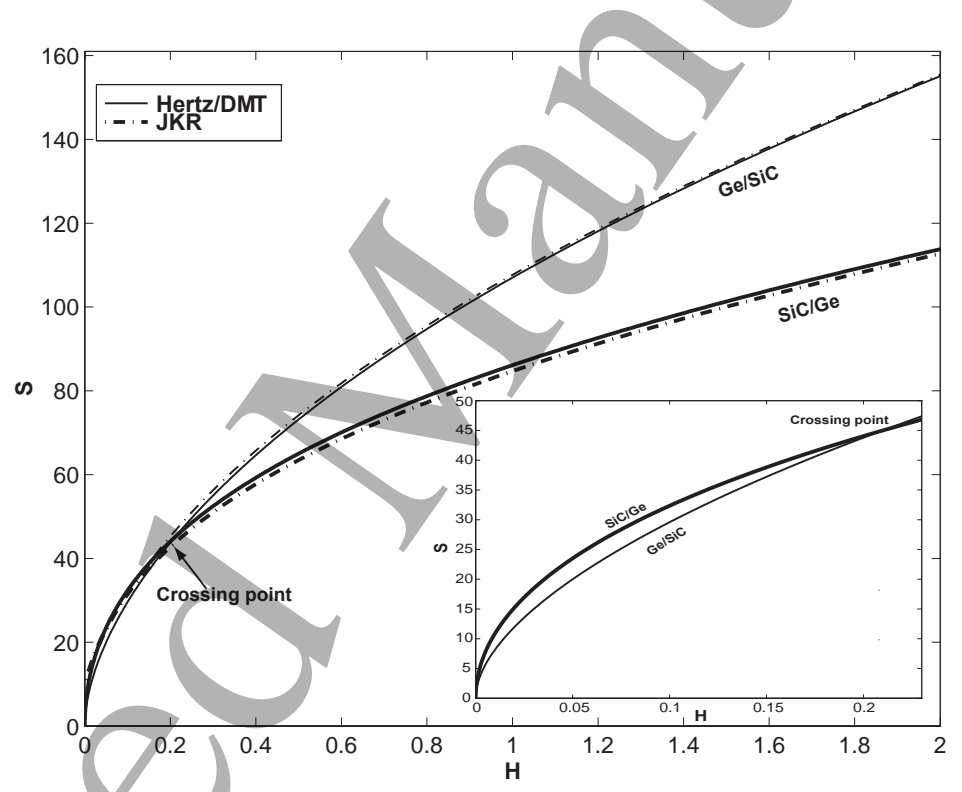

Figure 2. The $H-S$ curves of two bilayer structures: Ge/SiC (softer film/harder substrate) and $\mathrm{SiC} / \mathrm{Ge}$ (harder film/softer substrate). The dashed lines are the ones predicted by the JKR model and solid ones are those by the Hertz or DMT model. The inset plot shows a closer look at the contact stiffness at small indentation depths. The contact stiffness curves of $\mathrm{Ge} / \mathrm{SiC}$ and $\mathrm{SiC} / \mathrm{Ge}$ cross each other around $H=0.2$. 
Figure 3 The $H-f$ curves of $\mathrm{Ge} / \mathrm{SiC}$ and $\mathrm{SiC} / \mathrm{Ge}$. Here $f=E^{\prime} / E_{i i}^{\prime}=\alpha_{3}+\left(\alpha_{2}-\alpha_{3}\right) \phi(x)$ is the (dimensionless) effective reduced Young's modulús of the film/substrate bilayer structure. The two curves intersect around $H=0.2$.

\subsection{Detecting the subsurface layer properties}

To graphically illustrate how the inverse problem is solved, a special case is presented, in which the film reduced modulus $\left(\alpha_{2}\right)$ is known, the substrate reduced modulus $\left(\alpha_{3}\right)$ and the film thickness $(T)$ are the two unknowns. Physically, this is an inverse problem of detecting the properties of subsurface layer. In many applications of detecting the surface properties, measuring the thickness of surface layer (film layer) can be difficult or even impossible. For Ge/SiC, $\alpha_{2}=1.6239$; the $H-S$ curve as presented in figure 2 can be obtained from the indentation test. Here the DMT model is applied [32] and the film thickness is $T=2$. From Eqs. (11) and (12), $A$ and $\alpha_{1}$ can be extracted from the $H-S$ curve as $A=\sqrt{H / \beta_{1}}$ and $\alpha_{1}=2 \beta_{1}^{3 / 2} S /\left(3 H^{1 / 2}\right)$. Two (arbitrary) sets of data are thus extracted from the DMT $H-S$ curve of figure 2 as follows: Shallow indentation of $\left(\alpha_{1}, A, H\right)=(1.2571,2.4,0.4022)$ and deep indentation of $\left(\alpha_{1}, A, H,\right)=(1.3367,4.64,1.5032)$. According to Eq. (13), the corresponding effective reduced modulus 
of $\mathrm{Ge} / \mathrm{SiC}$ at shallow indentation is $f=2 \alpha_{1} /\left(2-\alpha_{1}\right)=3.3842$. For given $\alpha_{2}=1.6239$ and $A=2.4$ (or $H=0.4022$ ), $f$ as a function of both $\alpha_{3}$ and $T$ is plotted by Eq. (14) in figure 4. The horizontal plane in figure 4 is the one with the fixed value of $f=3.3842$ and intersection of the two planes are marked by a solid line. Physically, this intersection line means that for given $\alpha_{2}=1.6239$ and $A=2.4$, there are infinite combinations of $\alpha_{3}$ and $T$ which can yield the same value of $f=3.3842$. For the deep indentation, the corresponding reduced modulus of the film/substrate is $f=4.0305$. Similarly, for given $\alpha_{2}=1.6239$ and $A=4.64$ (or $H=1.5032$ ), $f$ as a function of both $\alpha_{3}$ and $T$ is plotted in figure 5 . In figure 5 , the horizontal plane is the one with the fixed value of $f=4.0305$ and intersection of the two planes are marked by a dashed line. Again, this intersection line/means that for given $\alpha_{2}=1.6239$ and $A=4.64$, there are infinite combinations of $\alpha_{3}$ and $T$ which can yield the same effective reduced modulus of $f=4.0305$. When these two curves obtained in figures 4 and 5 are projected into the $\alpha_{3}-T$ plane as shown in figure 6 , the intersection of the two curves is marked with a circle, which corresponds to the exact solution of $\left(\alpha_{3}, T\right)=(4.4764,2)$. The intersection mechanism for these two curves, or say, the mechanism that the inverse problem can be solved is reflected in Eq. (14): at different indentation depth $(H)$, the impacts of the film thickness $(T)$ and the substrate reduced modulus $\left(\alpha_{3}\right)$ on the reduced modulus of the film/substrate bilayer $(f)$ are different.

The above graphical solution procedure can be more succinctly presented by using Eq. (15). With $\alpha_{2}=1.6239$ and two sets of indentation data of $\left(\alpha_{1}, A, H\right)=(1.2571,2.4,0.4022)$ and $\left(\alpha_{1}, A, H\right)=$ $(1.3367,4.64,1.5032)$ as inputs, the following equation set is obtained

$$
\left\{\begin{array}{l}
\mathcal{F}\left(\alpha_{1}, A, \alpha_{2}, \alpha_{3}, T\right)=\mathcal{F}\left(1.2571,2.4,1.6239, \alpha_{3}, T\right)=\frac{2 \times 1.2571}{2-1.2571}-\alpha_{3}-\left(1.6239-\alpha_{3}\right) \phi\left(\frac{2.4}{T}\right)=0 \\
\mathcal{F}\left(\alpha_{1}, A, \alpha_{2}, \alpha_{3}, T\right)=\mathcal{F}\left(1.3367,4.64,1.6239, \alpha_{3}, T\right)=\frac{2 \times 1.3367}{2-1.3367}-\alpha_{3}-\left(1.6239-\alpha_{3}\right) \phi\left(\frac{4.64}{T}\right)=0 .
\end{array}\right.
$$

Eq. (16) provides two nonlinear equations for solving the two unknowns of $\alpha_{3}$ and $T$. The two unknowns are solved by the Newton-Raphson method [46], which yields the exact solution of $\left(\alpha_{3}, T\right)=(4.4764,2)$. 
Figure 4. The plot of $f$ as a function of $T$ and $\alpha_{3}$. The horizontal plane is the one with the fixed value of $f=3.3842$. The intersection of the two planes are marked by a solid line.

Theoretically, an $H-S$ curve obtained by an indentation test can provide infinite data set of $\left(\alpha_{1}, A, H\right)$. Here $\alpha_{3}$ and $T$ are the only two unknowns to be determined. Therefore, mathematically, only two different sets of indentation data are needed for solving the inverse problem. If two different sets of $\left(\alpha_{1}, A, H\right)=$ $\left(0.9592,0.2,2.7927 \times 10^{-3}\right)$ and $(1.3,3.3,0.7603)$ from the DMT $H-S$ curve of figure 2 are (arbitrarily) taken as the inputs, the following equation set is obtained

$$
\left\{\begin{array}{l}
\mathcal{F}\left(\alpha_{1}, A, \alpha_{2}, \alpha_{3}, T\right)=\mathcal{F}\left(0.9592,0.2,1.6239, \alpha_{3}, T\right)=\frac{2 \times 0.9592}{2-0.9592}-\alpha_{3}-\left(1.6239-\alpha_{3}\right) \phi\left(\frac{0.2}{T}\right)=0 \\
\mathcal{F}\left(\alpha_{1}, A, \alpha_{2}, \alpha_{3}, T\right)=\mathcal{F}\left(1.3,3.3,1.6239, \alpha_{3}, T\right)=\frac{2 \times 1.3}{2-1.3}-\alpha_{3}-\left(1.6239-\alpha_{3}\right) \phi\left(\frac{3.3}{T}\right)=0 .
\end{array}\right.
$$

Again, the exact solution of $\left(\alpha_{3}, T\right)=(4.4764,2)$ is obtained. In both Eqs. (16) and (17), the input data are the exact ones às obtained from the $H-S$ curves. As far as two different sets of $\left(\alpha_{1}, A, H\right)$ are input, the exact solution of $\left(\alpha_{3}, T\right)$ can be obtained. When applying the Newton-Raphson method [46], the initial guesses for $\alpha_{3}$ and $T$ need to be provided. One of the advantages for the above inverse problem formulation is that it is not sensitive to the initial guesses of $\alpha_{3}$ and $T$. However, in a real indentation experiment, experimental data are always with errors. As mentioned above, the assumption 


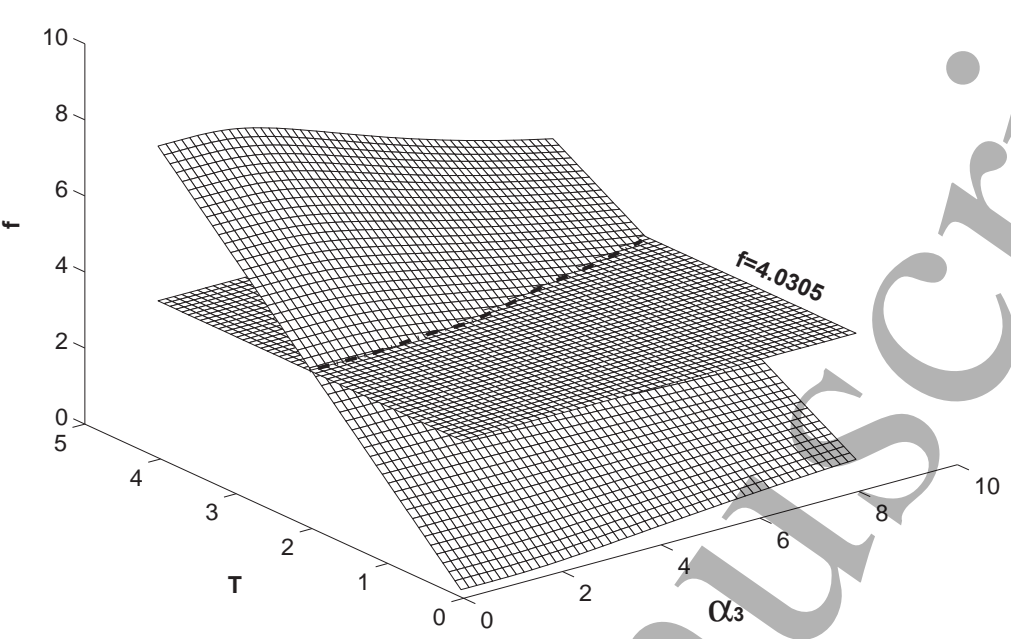

Figure 5. The plot of $f$ as a function of $T$ and $\alpha_{3}$. The horizontal plane is the one with the fixed value of $f=4.0305$. The intersection of the two planes are marked by a dashed line.

of $h=h_{c}$ is an important error source. Once the input data are with errors, the above conculsion of two different sets of $\left(\alpha_{1}, A, H\right)$ resulting in the same solution of $\left(\alpha_{3}, T\right)$ cannot hold. The inverse problem becomes overdetermined [44], i.e., different inputs will yield different solutions of $\left(\alpha_{3}, T\right)$. The robustness or sensitivity [44] is an issue in the inverse problem solving method. Here the robustness is studied by inputting the (arbitrarily) errored data.

The two exact data as given in Eq. (16) are $\left(\alpha_{1}, A\right)=(1.2571,2.4)$ and $(1.3367,4.64)$. The two errored data sets are taken as $\left(\alpha_{1}, A\right)=(1.2571 \times(1-1 \%), 2.4 \times(1+10 \%))=(1.2445,2.64)$ and $(1.3367 \times(1-$ $0.5 \%), 4.64 \times(1+5 \%))=(1.33,4.872)$. With the two errored data sets as input, Eq. (16) now becomes the following

$$
\left\{\begin{array}{l}
\mathcal{F}\left(\alpha_{1}, A, \alpha_{2}, \alpha_{3}, T\right)=\mathcal{F}\left(1.2445,2.64,1.6239, \alpha_{3}, T\right)=\frac{2 \times 1.2445}{2-1.2445}-\alpha_{3}-\left(1.6239-\alpha_{3}\right) \phi\left(\frac{2.64}{T}\right)=0 \\
\mathcal{F}\left(\alpha_{1}, A, \alpha_{2}, \alpha_{3}, T\right)=\mathcal{F}\left(1.33,4.872,1.6239, \alpha_{3}, T\right)=\frac{2 \times 1.33}{2-1.33}-\alpha_{3}-\left(1.6239-\alpha_{3}\right) \phi\left(\frac{4.872}{T}\right)=0 .
\end{array}\right.
$$

With the errored inputs, Eq. (18) yields the solution of $\left(\alpha_{3}, T\right)=(4.6804,2.6701)$. Compared with the exact solution of $\left(\alpha_{3}, T\right)=(4.4764,2)$, the errors of $\alpha_{3}$ and $T$ are $4.558 \%$ and $33.504 \%$, respectively. 
2

3

4

5

6

7

8

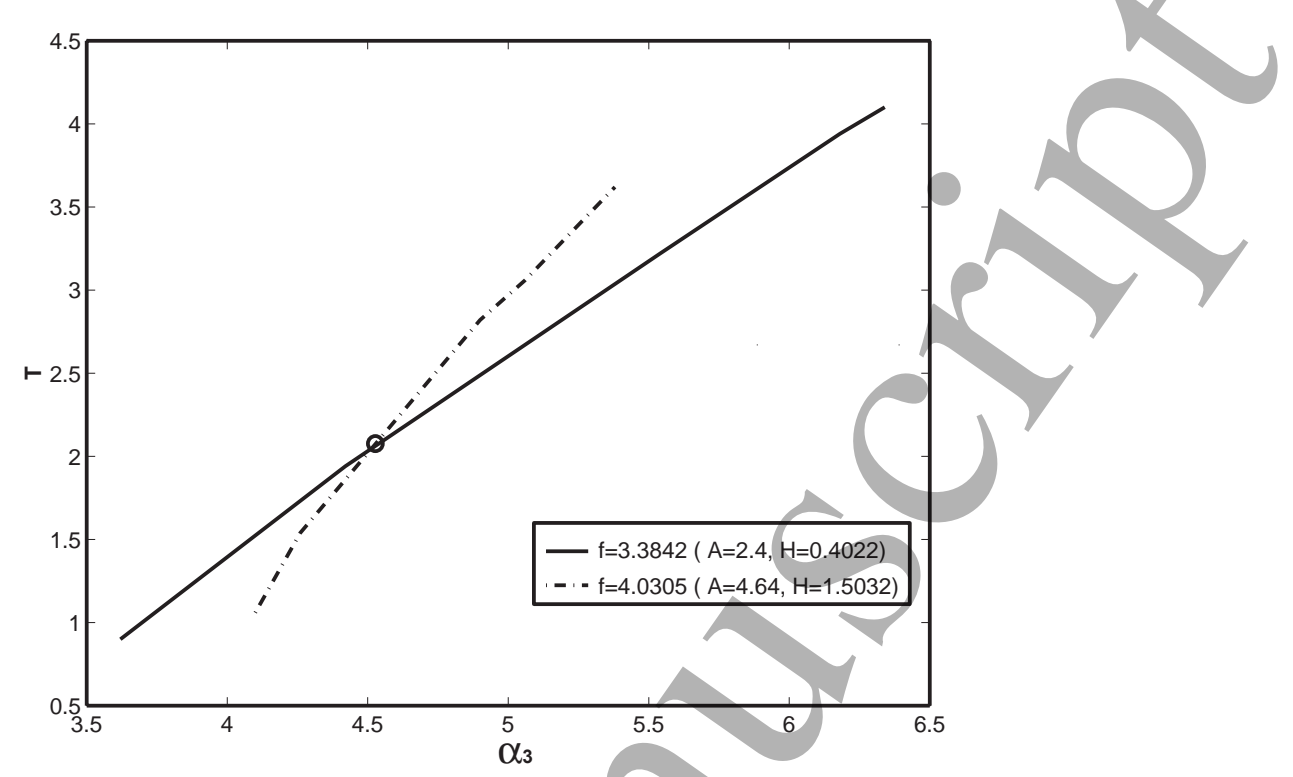

Figure 6. The two intersection curves as obtained in Figs. 4 and 5 are projected into the $\alpha_{3}-T$ plane. The intersection of the two curves is marked by a circle, which corresponds to the exact solution of $\left(\alpha_{3}, T\right)=(4.4764,2)$.

Further robustness study are carried out by using dozens of errored data as inputs, which, for the sake of succinctness, are not presented here. The general trend is clear: the above inverse solving method is extremely sensitive to the $\alpha_{1}$ error. Small $\alpha_{1}$ error can result in large errors of $\alpha_{3}$ and $T$, and even a worse scenario that the Newton-Raphson method cannot find a solution. As shown later, this robustness issue becomes more serious when there are more unknowns in the inverse problem.

\subsection{Detecting the properties of both film and substrate layers}

Now $\alpha_{2}, \alpha_{3}$ and $T$ are three unknowns. To solve the three unknowns, total three sets of data are needed. As the three sets of $\left(\alpha_{1}, A\right)=(1.2571,2.4),(1.3,3.3)$ and $(1.3367,4.64)$ are supplied, Eq. (15) yields the 
following equation set

$$
\left\{\begin{array}{l}
\mathcal{F}\left(\alpha_{1}, A, \alpha_{2}, \alpha_{3}, T\right)=\mathcal{F}\left(1.2571,2.4, \alpha_{2}, \alpha_{3}, T\right)=\frac{2 \times 1.2571}{2-1.2571}-\alpha_{3}-\left(\alpha_{2}-\alpha_{3}\right) \phi\left(\frac{2.4}{T}\right)=0 \\
\mathcal{F}\left(\alpha_{1}, A, \alpha_{2}, \alpha_{3}, T\right)=\mathcal{F}\left(1.3,3.3, \alpha_{2}, \alpha_{3}, T\right)=\frac{2 \times 1.3}{2-1.3}-\alpha_{3}-\left(\alpha_{2}-\alpha_{3}\right) \phi\left(\frac{3.3}{T}\right)=0 \\
\mathcal{F}\left(\alpha_{1}, A, \alpha_{2}, \alpha_{3}, T\right)=\mathcal{F}\left(1.3367,4.64, \alpha_{2}, \alpha_{3}, T\right)=\frac{2 \times 1.3367}{2-1.3367}-\alpha_{3}-\left(\alpha_{2}-\alpha_{3}\right) \phi\left(\frac{4.64}{T}\right)=0 .
\end{array}\right.
$$

Again, as far as the exact data are supplied, the Newton-Raphson method is not sensitive to the initial guesses of $\alpha_{2}, \alpha_{3}$ and $T$ and their exact solutions are also obtained. Now the robustness issue is studied by inputting the three sets of errored data: $\left(\alpha_{1}, A\right)=(1.2571 \times(1-1 \%), 2.4 \times(1+10 \%))=(1.2445,2.64)$, $(1.33 \times(1-0.7 \%), 3.3 \times(1+5 \%))=(1.3207,3.465)$ and $(1.3367 \times(1-0.1 \%), 4.64 \times(1+3 \%))=$ (1.3354, 4.7792). With the errored inputs, Eq. (15) leads to the following equation set

$$
\left\{\begin{array}{l}
\mathcal{F}\left(\alpha_{1}, A, \alpha_{2}, \alpha_{3}, T\right)=\mathcal{F}\left(1.2445,2.64, \alpha_{2}, \alpha_{3}, T\right)=\frac{2 \times 1.2445}{2-1.2445}-\alpha_{3}-\left(\alpha_{2}-\alpha_{3}\right) \phi\left(\frac{2.64}{T}\right)=0 \\
\mathcal{F}\left(\alpha_{1}, A, \alpha_{2}, \alpha_{3}, T\right)=\mathcal{F}\left(1.3207,3.465, \alpha_{2}, \alpha_{3}, T\right)=\frac{2 \times 1.3207}{2-1.3207}-\alpha_{3}-\left(\alpha_{2}-\alpha_{3}\right) \phi\left(\frac{3.465}{T}\right)=0 \\
\mathcal{F}\left(\alpha_{1}, A, \alpha_{2}, \alpha_{3}, T\right)=\mathcal{F}\left(1.3354,4.7792, \alpha_{2}, \alpha_{3}, T\right)=\frac{2 \times 1.3354}{2-1.3354}-\alpha_{3}-\left(\alpha_{2}-\alpha_{3}\right) \phi\left(\frac{4.7792}{T}\right)=0 .
\end{array}\right.
$$

Eq. (20) yields the solution of $\left(\alpha_{2}, \alpha_{3}, T\right)=(1.1524,4.6407,2.2181)$. Compared with the exact solution of $\left(\alpha_{2}, \alpha_{3}, T\right)=(1.6239,4.4764,2)$, the errors of $\alpha_{2}, \alpha_{3}$ and $T$ are $-29.03 \%, 3.67 \%$ and $10.91 \%$, respectively. With one more unknown, the Newton-Raphson method becomes much more sensitive to the $\alpha_{1}$ errors. Furthermore, it becomes much more difficult for the Newton-Raphson method to find the solutions unless the $\alpha_{1}$ errors are small and in certain range. The physical mechanism for the inverse problem to be solved is that the film thickness $(T)$ and the reduced moduli of film and substrate $\left(\alpha_{2}\right.$ and $\left.\alpha_{3}\right)$ play different roles in determining the effective reduced modulus of the film/substrate bilayer $(f)$ at different indentation depth. The formulation of the inverse problem as presented in Eq. (19) is a straightforward one. However, the robustness is a serious issue here. For the above inverse problem solving method to work, it requires the supplied data with very high accuracy, which can be extremely challenging in some indentation tests. This robustness or sensitivity issue is often encountered in solving an inverse problem 
because of its overdetermined and ill-posed properties [44].

To circumvent the robustness issue, the $\alpha_{2}, \alpha_{3}$ and $T$ impacts on the contact stiffness need to be systematically studied. Figure 7 presents the film thickness $(T)$ influence on the $H-S$. In figure 7 , there are two groups of the bilayer structures: harder film/softer substrate $(\mathrm{SiC} / \mathrm{Ge})$ and softer film/harder substrate $(\mathrm{Ge} / \mathrm{SiC})$. The crossing of the $H-S$ curves of these two types of bilayen structures is studied in figure 2, which occurs in relatively shallow indentation area. Now the focus is on the relatively deep indentation area, say, $H>0.5$. There is an obvious trend as seen in figure 7: At a given indentation depth $H$, the contact stiffness of harder film $(\mathrm{SiC} / \mathrm{Ge})$ monotonically increase with the increase of $T$. In contrast, it is a monotonical decrease of the contact stiffness of softer film $(\mathrm{Ge} / \mathrm{SiC})$. The mechanism is the following: As seen in Eq. (14), increasing the film thickness of a harder film increases $f$, which thus leads to the increase of harder film contact stiffness. Similarly, for a softer film, increasing the film thickness effectively reduces $f$ and thus causes the decrease of its contact stiffness. The "bending upwards" behavior of $\mathrm{SiC} / \mathrm{Ge}$ and the "bending downwards" behavior of Ge/SiC with the $T$ increase are a benchmark difference between the harder film/softer substrate and softer film/harder substrate bilayer structures. This benchmark difference is also noticed by Li and Chou [47] in a different way of examining the indenter surface displacement.

Figure 8 presents the combined effects of $\alpha_{2}, \alpha_{3}$ and $T$ on the contact stiffness. There are five contact stiffness curves in figure 8, $S_{1}$ is the $H-S$ curve for the $\mathrm{Ge} / \mathrm{SiC}$ with $\left(\alpha_{2}, \alpha_{3}, T\right)=(1.6239,4.4764,2)$. Except for $S_{1}$ of $\mathrm{Ge} / \mathrm{SiC}$ with the film thickness of $T=2$, the other four are with $T=3$. $S_{2}$ is with $\left(\alpha_{2}, \alpha_{3}\right)=(3,7)$. As the $\alpha_{2}$ and $\alpha_{3}$ of $S_{2}$ are both larger than those of $S_{1}, S_{2}$ is referred to as the larger/larger case. $S_{3}$ is with $\left(\alpha_{2}, \alpha_{3}\right)=(1,7)$. Similarly, as $\alpha_{2}$ of $S_{3}$ is smaller than that of $S_{1}$ and $\alpha_{3}$ of $S_{3}$ is larger than that of $S_{1}$, it is referred to as the smaller/larger case. $S_{4}$ is with $\left(\alpha_{2}, \alpha_{3}\right)=(3,2)$, which is referred to as the larger/smaller case. $S_{5}$ is with $\left(\alpha_{2}, \alpha_{3}\right)=(1,2)$ and is referred to as the 
Figure 7. The $H-S$ curves of the harder film (SiC/Ge) and the softer film $(\mathrm{Ge} / \mathrm{SiC})$. As the film thickness $(T)$ increases, the contact stiffness of the harder film monotonically increases and in contrast, that of the softer film monotonically decreases.

smaller/smaller case. In the deep indentation area $(H \geq 0.5)$, the contact stiffnesses of $S_{2}$ and $S_{3}$ are larger than that of $S_{1}$ and in comparison, those of $S_{4}$ and $S_{5}$ are smaller than that of $S_{1}$. As the indentation depth progresses, the reduced modulus of the film/substrate, $f$, rapidly approaches the substrate modulus $\left(\alpha_{3}\right)$. Both $S_{2}$ and $S_{3}$ have larger $\alpha_{3}$, which is responsible for their larger contact stiffness than that of $S_{1}$ in the deep indentation area. Similarly, because the $\alpha_{3}$ of $S_{4}$ and $S_{5}$ is smaller than that of $S_{1}$, it leads to the smaller contact stiffness in the deep indentation area. The inset of figure 8 shows the $H-S$ in shallow indentation area. It is noticed that $S_{1}$ is crossed by two curves of $S_{3}$ and $S_{4}$. The crossing mechanism is the same as that discussed in figure 2: For the smaller/larger case of $S_{3}$, the smaller film modulus $\left(\alpha_{2}\right)$ plays dominant role in $f$ at shallow indentation, which results in a smaller contact stiffness than that of $S_{1}$. While, at deep indentation, its larger substrate modulus $\left(\alpha_{3}\right)$ is the dominant one which is responsible for its larger contact stiffness. In summary, for the smaller/larger case of $S_{3}$, 
Figure 8. The Young's modulus impact on the contact stiffness. $\mathrm{S}_{1}$ is with $T=2$ and all others are with $T=3 . \mathrm{S}_{1}$ is the $\mathrm{Ge} / \mathrm{SiC}$ with $\alpha_{2}=1.6239$ and $\alpha_{3}=4.4764 ; \mathrm{S}_{2}$ is the larger/larger case with $\alpha_{2}=3$ and $\alpha_{3}=7 ; \mathrm{S}_{3}$ is the smaller/larger case with $\alpha_{2}=1$ and $\alpha_{3}=7 ; \mathrm{S}_{4}$ is the larger/smaller case with $\alpha_{2}=3$ and $\alpha_{3}=2 ; \mathrm{S}_{5}$ is the larger/larger case with $\alpha_{2}=1$ and $\alpha_{3}=2$. The inset is a closer look at the small indentation depth, which shows the crossing of the $\mathrm{S}_{2}$ and $\mathrm{S}_{3}$ over $\mathrm{S}_{1}$.

its contact stiffness experiences the smaller and then larger transition as the indentation depth increases, which causes the crossing. Similarly, the larger/smaller case of $S_{4}$ experiences the larger and then smaller transition of its contact stiffness, which aslo leads to a crossing. For the larger/larger cases of $S_{2}$, it always has larger effective modulus of $f$ and thus larger contact stiffness. Therefore, there is no crossing. Similarly, the smaller/smaller case of $S_{5}$ always has smaller effective modulus of $f$ and contact stiffness and thus no crossing. It is also noticed that the contact stiffness differences between $S_{1}$ and other curves enlarge as the contact depth increases. These enlarging differences are more directly illustrated in figure 9. In the deep indentation area as seen in figure $9, S_{2}-S_{1}$ and $S_{3}-S_{1}$ increase as $H$ increases; $S_{4}-S_{1}$ and $S_{5}-S_{1}$ decrease as $H$ increases; both result in the enlarging difference of contact stiffness. Here we 
need to address the mechanism for the enlarging difference of contact stiffness, which is key to finding the substrate modulus $\alpha_{3}$. As defined in Eq. (10), $\alpha_{1}$ is the dimensionless modulus of the bilayer/indenter system and is related with $f$ by Eq. (13) as follows

$$
\alpha_{1}=\frac{2 f}{2+f}=\frac{2}{2 / f+1}
$$

As presented in figue 3 , the reduced modulus of the film/substrate $(f)$ starts with the film modulus $\alpha_{2}$ and approaches the substrate modulus $\alpha_{3}$ as $H$ increases. Because $f$ is a monotonical function, $\alpha_{1}$ monotonically approaches $2 \alpha_{3} /\left(2+\alpha_{3}\right)$ in deep indentation area as given by Eq. (21). According to the DMT model of Eq. (12), $S \propto \alpha_{1} \sqrt{H}$, which leads to $S \propto 2 \alpha_{3} /\left(2+\alpha_{3}\right) \sqrt{H}$ in deep indentation area. Therefore, $S_{2}-S_{1} \propto\left[2 \alpha_{3}^{2} /\left(2+\alpha_{3}^{2}\right)-2 \alpha_{3}^{1} /\left(2+\alpha_{3}^{1}\right)\right] \sqrt{H}$ in deep indentation area. Here $\alpha_{3}^{2}=7$ is the substrate modulus of $S_{2} ; \alpha_{3}^{1}=4.4764$ is the substrate modulus of Ge/SiC bilayer of $S_{1}$. In this study, $\alpha_{3}^{i}$ stand for the substrate modulus of the $S_{i}$ curve. Because $\alpha_{3}^{2}>\alpha_{3}^{1}, S_{2}-S_{1}$ is a monotonically increasing function of $H$. Similarly, because $\alpha_{3}^{5}<\alpha_{3}^{1}, S_{5}-S_{1}$ is a monotonically decreasing function of $H$. In figure 9 , it is also noticed that in contrast to the enlarging differences of $S_{2}-S_{1}, S_{3}-S_{1}, S_{4}-S_{1}$ and $S_{5}-S_{1}$, the slopes of $S_{3}-S_{2}$ and $S_{5}-S_{4}$ approaches zero in deep indentation area. The reason is simple: The substrate moduli of $S_{2}$ and $S_{3}$ are the same as $\alpha_{3}^{2}=\alpha_{3}^{3}=7$; the substrate moduli of $S_{4}$ and $S_{5}$ are the same as $\alpha_{3}^{4}=\alpha_{3}^{5}=2$. Therefore, because they share the same substrate modulus, their contact stiffness difference remains unchanged in deep indentation area. Actually, the same mechanism also works for those curves in figure 7. As seen in figure 7, the three curves of the $\mathrm{Ge} / \mathrm{SiC}$ are "parallel" to each other in deep indentation area; the three/curves of the $\mathrm{SiC} / \mathrm{Ge}$ are also "parallel" to each other. The influence of the film modulus and thickness on the contact stiffness is confined in relatively shallow indentation area. The substrate modulus is the only dominant factor influencing the contact stiffness in deep indentation area, which is the mechanism for us to determine the substrate modulus of $\alpha_{3}$ [45]. Suppose that $S_{1}$ is the experimental data of indentation test; $\alpha_{3}^{1}$ is its substrate modulus; $S_{g}$ is the guessed $H-S$ curve with 
the substrate modulus of $\alpha_{3}^{g}$. In deep indentation area, if $S_{g}-S_{1}$ is a monotonically decreasing function, $\alpha_{3}^{g}<\alpha_{3}^{1}$; if $S_{g}-S_{1}$ is a monotonically increasing function, $\alpha_{3}^{g}>\alpha_{3}^{1}$; if $S_{g}-S_{1}$ remains constant or varies very slowly (for the real application purpose), $\alpha_{3}^{g}=\alpha_{3}^{1}$ or $\alpha_{3}^{g}$ is very close to $\alpha_{3}^{1}$. This is an easy and straight forward method of determining $\alpha_{3}$.

Once $\alpha_{3}$ is found by the data of deep indentation, the next goal is to find the filmmodulus $\left(\alpha_{2}\right)$ and

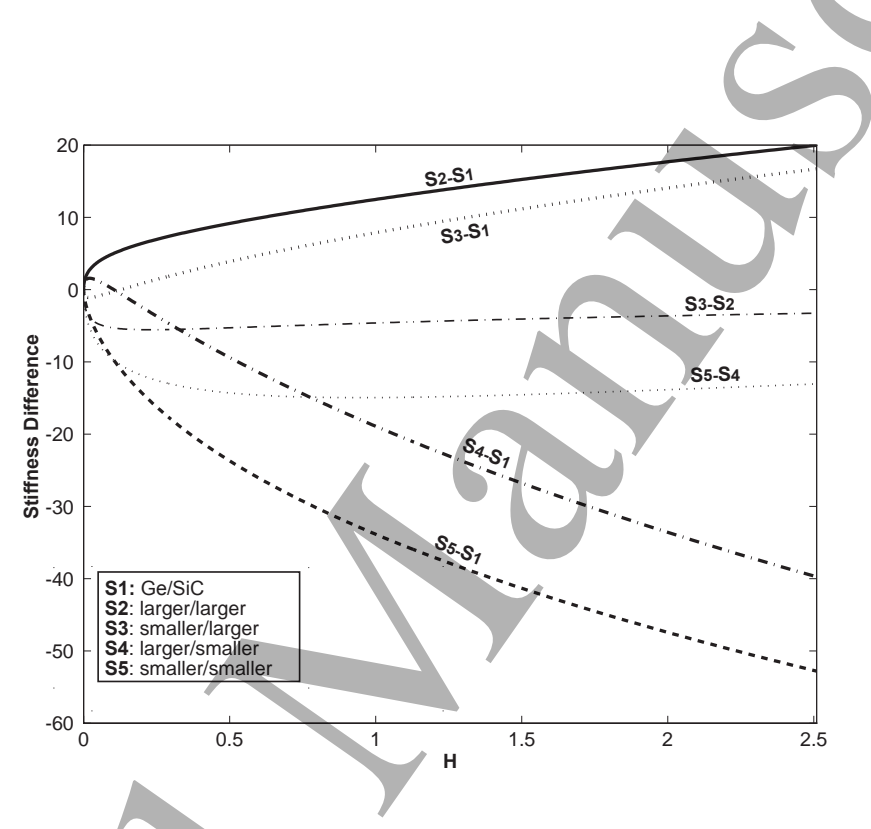

Figure 9. The contact stiffness difference as a function of $H$.

thickness $(T)$ in shallow indentation area. Suppose the that $\mathrm{Ge} / \mathrm{SiC}$ is the target bilayer structure and its parameters of $\alpha_{2}^{t}=1.6239, \alpha_{3}^{t}=4.4764$ and $T^{t}=2$ are the unknown parameters to be determined by its $H-S$ curve obtained from experiment. Here the superscript $t$ stands for "target". By examining the data of deep indentation and using the approach outlined above, $\alpha_{3}=4.4764$ can be found and all the curves in figure 10 are with $\alpha_{3}^{t}=4.4764$. The $H-S$ curve of the monolithic $\mathrm{SiC}$, i.e., $\alpha_{2}=\alpha_{3}=4.4764$, is plotted in figure 10. Because the $H-S$ curve of the target bilayer structure is always below that of the monolithic $\mathrm{SiC}$, it can be told that it is the case of the softer film on harder substrate, i.e., $\alpha_{2}<\alpha_{3}$, which also effectively confine the $\alpha_{2}$ range. Firstly, we start to guess a very small film modulus of $\alpha_{2}=1$. The 
Figure 10. The contact stiffness of softer film at small indentation depths. The "bending downwards" behavior as indicated by the two arrows is noticed. The solid lines are the Ge/SiC bilayer and monolithic SiC layer.

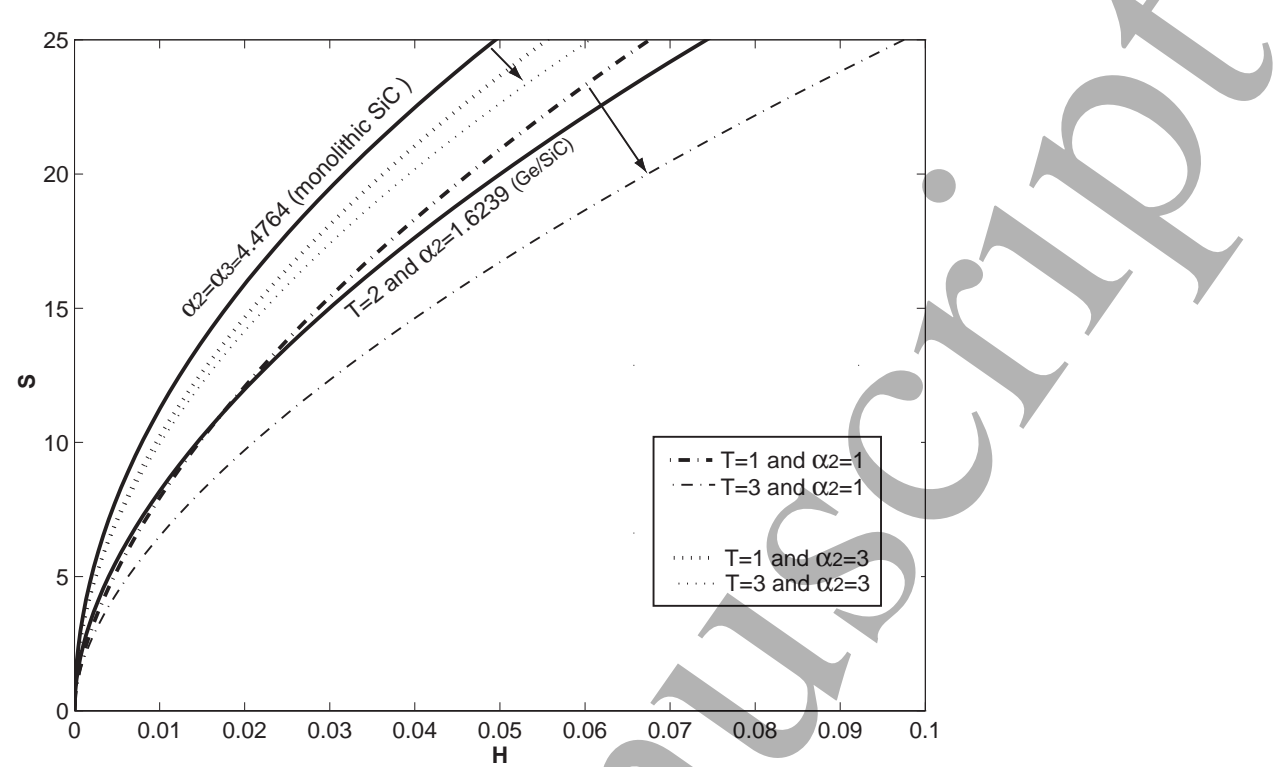

two curves of $\alpha_{2}=1$ with two different $T=1$ and $T=3$ are plotted in figure 10 . It is noticed that the curve of $\alpha_{2}=1$ and $T=1$ crosses that of the target bilayer. Before the crossing, the contact stiffness with $\alpha_{2}=1$ and $T=1$ is smaller than that of the target bilayer, we can thus tell $\alpha_{2}^{t}>1$ because at the very shallow indentation, the film modulus is the dominant impact factor on the contact stiffness. After the crossing, the contact stiffness of the target bilayer is smaller than that with $\alpha_{2}=1$ and $T=1$ and we can tell that $T^{t}>1$. The reasons are the following: the target bilayer is the softer film on harder substrate and $\alpha_{2}^{t}>1$. The only choice for the contact stiffness with $\alpha_{2}=1$ and $T=1$ to surpass that of the target bilayer is that the target bilayer has a larger film thickness. Because of the larger film thickness, the effective modulus $f$ of the target bilayer approaches the substrate harder modulus of $\alpha_{3}=4.4764$ at a much slower rate, which results in the smaller contact stiffness of the target bilayer after crossing. Compared with that of $\alpha_{2}=1$ and $T=1$, the $H-S$ curve of $\alpha_{2}=1$ and $T=3$ "bends downwards" as 
Figure 11. The contact stiffness of harder film at small indentation depths. The "bending upwards" behavior as indicated by the two arrows is noticed. The solid lines are the $\mathrm{SiC} / \mathrm{Ge}$ bilayer and monolithic Ge layer.

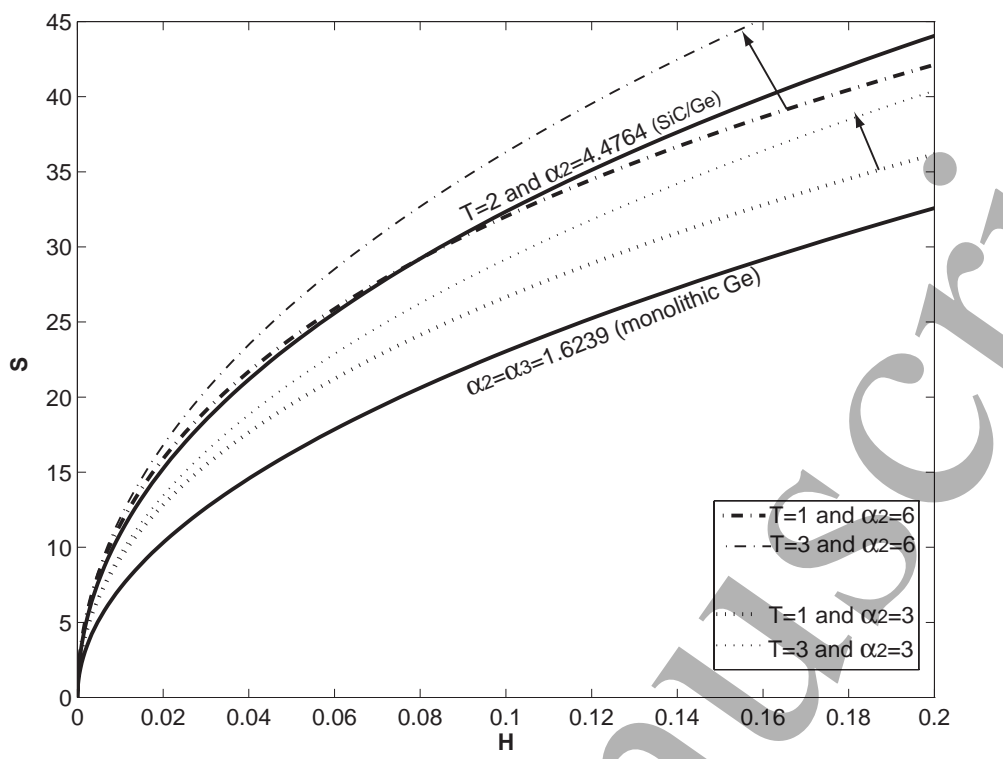

the film thickness increases, which is the characteristics of the softer film on harder substrate as discussed in figure 7. It is also seen that there is no crossing and the contact stiffness of $\alpha_{2}=1$ and $T=3$ is always smaller than that of the target bilayer. We conclude that $T^{t}<3$. The reason is as follows: If $T^{t}>3$, the same crossing scenario of the curve of $\alpha_{2}=1$ and $T=1$ with that of the target bilayer will occur again. Another two curves of $\alpha_{2}=3$ with two different $T=1$ and $T=3$ are also plotted in figure 10. Again, the "bending downwards" curve of $\alpha_{2}=3$ and $T=3$ is due to the characteristics of the softer film on harder substrate. Knowing that $1<T^{t}<3$ and the two curves with $\alpha_{2}=3$ are always above that of the target bilayer as seen in figure 10 , we conclude that $\alpha_{2}^{t}<3$. Otherwise, there will be crossing of curves. In summary, by examining the two groups of contact stiffness with varying film modulus and thickness, we limit the material property range of the target bilayer as the following: $1<\alpha_{2}^{t}<3$ and $1<T^{t}<3$. The above procedure can be systematically carried out to further narrow down the range. For example, 


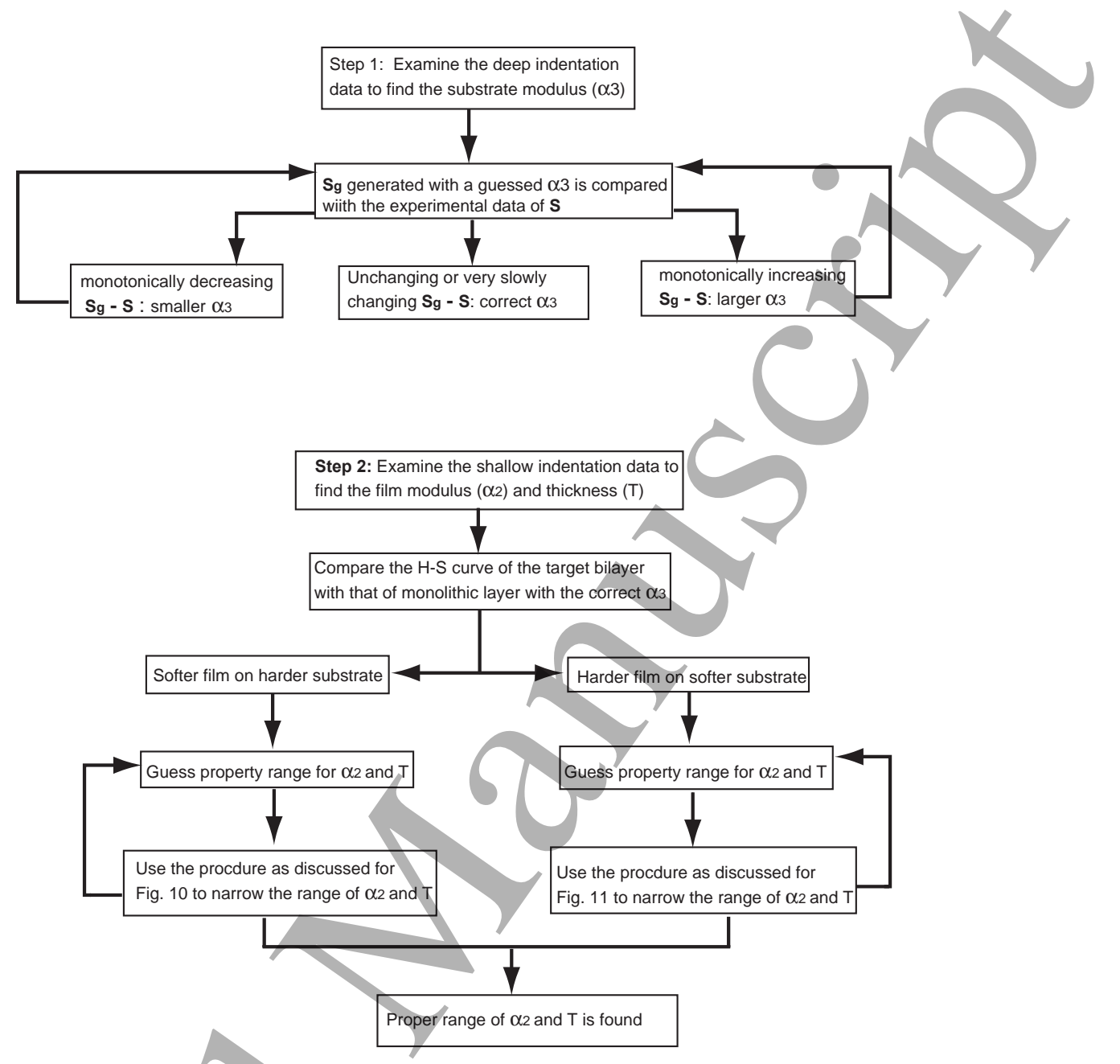

Figure 12. The flow charts of graphic solution for $\alpha_{2}^{t}, \alpha_{3}^{t}$ and $T^{t}$ by examining the $H-S$ curves.

with the above information, we can start the new procedure by guessing $\alpha_{2}^{t}=1.5$ and $2.5, T^{t}=1.5$ and 2.5 ; and repeat the above procedure until a satisfying range is obtained.

The above procedure is for the softer film on harder substrate case. For the harder film on softer substrate case, things are slightly different. We demonstrate the new procedure with the $\mathrm{SiC} / \mathrm{Ge}$ as the target bilayer. Now the parameters of $\alpha_{2}^{t}=4.4764, \alpha_{3}^{t}=1.6239$ and $T^{t}=2$ are the unknown parameters to be determined. Again, by examining the data of deep indentation and the approach as outlined in figures 8 and $9, \alpha_{3}^{t}=1.6239$ can be found. The $H-S$ curve of the monolithic Ge with $\alpha_{2}=\alpha_{3}=1.6239$ is plotted in figure 11. It is seen that the contact stiffness of monolithic Ge is always smaller than that 
of the target bilayer and we can tell that the bilayer is the harder film on softer substrate case with $\alpha_{2}^{t}>\alpha_{3}^{t}=1.6239$, which, again, gives us a range of the film modulus. In figure 11 , all $H-S$ curves are with $\alpha_{3}=1.6239$. We firstly guess a large film modulus. The two curves of $\alpha_{2}=6$ with $T=1$ and $T=3$ are firstly plotted. The $H-S$ curve with $\alpha_{2}=6$ and $T=1$ crosses that the target bilayer and as seen in figure 11, at very shallow indentation before the crossing, the contact stiffness of the target bilayer is smaller. Again, due to the dominant impact of the film modulus, we conclude $\alpha_{2}^{t}<6$ by examining the very shallow indentation data. Because of the crossing, we conclude $T^{t}>1$. The reason is that because of the the harder film on softer substrate case and $\alpha_{2}^{t}<6$, the only choice for the target bilayer with a larger contact stiffness after crossing is to have a large film thickness, which slows down target bilayer effective modulus $f$ transition to the softer substrate modulus of $\alpha_{3}$. The $H-S$ curve with $\alpha_{2}=6$ and $T=3$ is always above that of the target bilayer and we conclude $T^{t}<3$. The reason that if $T^{t}>3$, the crossing scenario as that of $\alpha_{2}=6$ and $T=1$ will occur. The two curves of $\alpha_{2}=3$ with $T=1$ and $T=3$ are also plotted and the two curves are always below that the target bilayer. In conjunction with $1<T^{t}<3$, we conclude $\alpha_{2}^{t}>3$. In summary, we can confine the material property range of the target bilayer as: $3<\alpha_{2}^{t}<6$ and $1<T^{t}<3$. Again, by repeating the above process, the material range can be further narrowed. In figure 11, the "bending upwards" behavior of the curves with $\alpha_{2}=6$ and $\alpha_{2}=3$ is noticed. Again, this behavior is to the characteristics of the harder film on softer substrate as discussed in figure 7.

The above graphic solution procedure for $\alpha_{2}^{t}, \alpha_{3}^{t}$ and $T^{t}$ is summarized in the flow charts of figure 12 . There are two main steps: Step one is to examine the deep indentation data to find $\alpha_{3}^{t}$; step two is to examine the shallow indentation data to refine the range of $\alpha_{2}^{t}$ and $T^{t}$. One characteristics of the bilayer indentation is that the film/substrate effective modulus $f$ rapidly approaches the substrate modulus $\left(\alpha_{3}\right)$ from the the film modulus $\left(\alpha_{2}\right)$ as the indentation depth increases. In the deep indentation area, $f$ is

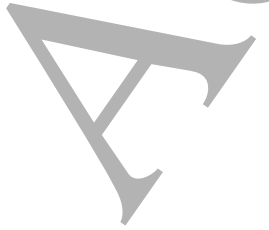


(close to) $\alpha_{3}^{t}$ and this property is used as an effective way of extracting the substrate modulus. In the rapid $f$ transition area of shallow indentation area, the contact stiffness shows the distinctive properties in the softer film on harder substrate and harder film on softer substrate cases as the film thickness increases, i.e., the "bending downwards" and the "bending upwards" behaviors, respectively. Those properties are utilized to systematically refine the range of $\alpha_{2}^{t}$ and $T^{t}$.

\section{Summary}

In many real applications of an indentation test, the film modulus, thickness and the substrate modulus of a bilayer composite structure are usually the three unknown parameters to determined. An inverse problem of solving the above three parameters is formulated and solved. The inverse problem formulation is a straightforward one, which directly compares the bilayer effective modulus as obtained by the experiment and the one predicted by a model. The physical mechanism that the inverse problem can be solved is due to the different roles of the three parameters playing in determining the bilayer effective modulus at different indentation depths. However, there is a robustness issue of solving the inverse problem, which requires very high accuracy for the experimental data of indentation tests. A graphical solution procedure, which utilizes the bilayer indentation properties in both deep and shallow indentation areas, is presented to circumvent the robustness issue and systematically refine the parameters' range.

\section{Acknowledgment}

The research has been supported by the National Natural Science Foundation of China (NSFC Nos. 11372321, 11772335 and 21203038), and by the Ministry of Science and Technology (MOST) of China (No. 2016YFA0200700). 


\section{References}

[1] Dielbold A C 2005 Science 310 61-2

[2] Shekhawat G S and Dravid V P 2005 Science 310 89-92

[3] Tetard L, Passian A, Venmar K T, Lynch R M, Voy B H, Shekhawat G S, Dravid V P and Thundat T 2008 Nat. Nanotech. 3 501-5

[4] Balke N, Jesse S, Morozovska A, Eliseeve E, Chung D W, Kim Y, Adamczyk L, Garcia R E, Dudney N J and Kalinin S V 2010 Nat. Nanotech. 5 749-54

[5] Balke N, Jesse S, Kim Y, Adamczyk L, Tselev A, Ivanov IN, Dudney NJ and Kalinin S V 2010 Nano Lett. $103420-5$

[6] Kolosov O and Yamanaka K 1993 Jpn. J. Appl. Phys. 32 L1095-L1098

[7] Rabe U and Arnold W 1994 Appl. Phys. Lett. 64 1493-5

[8] Cuberes M T, Assender H E, Briggs G A and Kolosov O 2000 J. Phys. D: Appl. Phys. 33 2347-55

[9] Martinez N F, Lozano J R, Heruzo E T, Garcia F, Richter C, Sulzbach T and Garcia R 2008 Nanotechnology 19384011

[10] Cantrell S A, Cantrell J H and Lillehei P T 2007 J. Appl. Phys. 101114324

[11] Tetard L, Passian A and Thundat T 2010 Nat. Nanotech. 5 105-9

[12] Garcia R, Magerle R and Perez R 2007 Nat. Mater. 6 405-11

[13] Yaralioglu G G, Degertekin F L, Crozier K B and Quate C F 2000 J. Appl. Phys. 87 7491-6 
[14] Hurley D C, Kopycinska-Müller M, Langlois E D, Kos A B and Barbosa N 2006 Appl. Phys. Lett. 84 449-51

[15] Batog G S, Baturin A S, Bormashov V S and Sheshin E P 2006 Tech. Phys. 51 1084-9

[16] Parlak Z and Degertekin F L 2008 J. Appl. Phys. 103114910

[17] Garcia R 2010 Nat. Nanotech. 5 101-2

[18] Hölscher H, Allers W, Schwarz U D, Schwarz A and Wiesendanger R 1999 Phys. Rev. Lett. 83 4780-3

[19] Stark M, Stark R W, Heckl W M and Guckenberger R 2000 Proc. Natl. Acad. Sci. U.S.A. 99 8473-8

[20] Lee M and Jhe W 2006 Phys. Rev. Lett. 97036104

[21] Menčik J, Munz D, Quandt E and Weppelmann E R 1997 J. Mater. Res. 12 2475-84

[22] Lim Y Y, Chaudhri M M and Enomoto Y 1999 J. Mater. Res. 14 2314-27

[23] Dimitriadis E K, Horkay F, Maresca J, Kachar B and Chadwick R 2002 Biophys. J. 82 2798-810

[24] Saha R and Nix W D 2002 Acta Mater. 50 23-38

[25] Li H and Vlassak J J 2009 J. Mater. Res. 24 1114-25

[26] Britnell L, Ribeiro RM, Eckamm A, Jalil R, Belle B D, Mishchenko A, Kim, YJ, Gorbachev R V, Georgiou S V, Morozov S V, Grigorenko A N, Geim A K, Casiraghi C, Castro Neto, A H and Novoselov K S 2013 Science 340 1311-4

[27] Hong X, Kim J, Shi S, Zhang Y, Jin C, Sun Y, Tongay S, Wu J, Zhang Y and Wang F 2014 Nat. Nanotech. 9 682-6 
[28] Bonaccorso F, Colombo L, Yu G, Stoller M, Tozzini V, Ferrari AC, Ruoff R and Pellegrini V 2015 Science $\mathbf{3 4 7} 1246501$

[29] Come J, Xie Y, Naguib M, Jesse S, Kalinin SV, Gogotsi Y, Kent P and Balke N 2016 Adv. Energy Mater. 61502290

[30] Lee C, Wei X, Kyser J W and Hone J 2008 Science 321 385-8

[31] Zhang Y 2016 Sci. China-Phys. Mech. Astron. 59624602

[32] Gao Y, Kim S, Zhou S, Chiu H, Nélias D, Berger C, de Heer W, Polloni L, Sordan R, Bongiorno A and Riedo E 2015 Nat. Mater. 14 714-20

[33] Bull S J 2005 J. Phys. D: Appl. Phys. 38, R293-R413

[34] Qu Z, Pei Y, He R and Fang D. 2016 J. Appl. Mech. 83041009

[35] Doerner M F and Nix W D 1986 J. Mater. Res. 1 601-9

[36] Pharr G M, Oliver W C and Brotzen F R 1992 J. Mater. Res. 7 613-7

[37] Hui C Y, Lin Y Y, Baney J M and Jagota A 2000 J. Adhesion Sci. Technol. 14 1297-319.

[38] Xu D, Ravi-Chandar K and Liechti K M 2008 J. Colloid Interface Sci. 318 507-19

[39] Oliver W C and Pharr G M 2004 J. Mater. Res. 19 3-20.

[40] Cheng Y and Cheng C 1998 Appl. Phys. Lett. 73, 614-6.

[41] Gao H, Chiu S and Lee J 1992 Int. J. Solids Struct. 29 2471-92.

[42] Vasu T S, Bhandakkar T K 2016 J. Appl. Mech. 83061009.

[43] Yu H Y, Sandy S C and Rath B B 1990 J. Mech. Phys. Solids 38 745-64. 
[44] Borodich D M and Galanov B A. 2008Proc. Roy. Soc. A 464 2759-76.

[45] Constantinescu A, Korsunsky A M, Pison O and Oueslati A. 2013 Int. J. Solids Struct. 50 2798-807.

[46] Press W H, Teukolsky S A, Vetterling W T and Flannery BP. 1992 Numerical Recipes in Fortran, 2nd edition, Cambridge, UK: Cambridge University Press.

[47] Li J and Chou T, 1997 Int. J. Solids Struct. 34, 4463-78. 